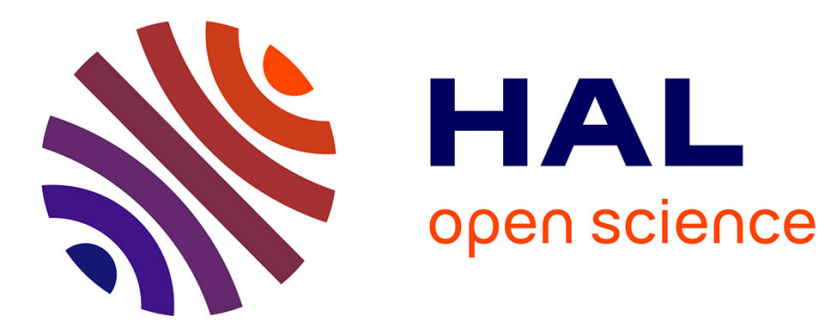

\title{
Two-dimensional features of correlations in the flow and near pressure fields of Mach number 0.9 jets \\ Christophe Bogey
}

\section{To cite this version:}

Christophe Bogey. Two-dimensional features of correlations in the flow and near pressure fields of Mach number 0.9 jets. 57th AIAA Aerospace Sciences Meeting, Jan 2019, San Diego, United States. 10.2514/6.2019-0806 . hal-02334578

\section{HAL Id: hal-02334578 \\ https://hal.science/hal-02334578}

Submitted on 26 Oct 2019

HAL is a multi-disciplinary open access archive for the deposit and dissemination of scientific research documents, whether they are published or not. The documents may come from teaching and research institutions in France or abroad, or from public or private research centers.
L'archive ouverte pluridisciplinaire HAL, est destinée au dépôt et à la diffusion de documents scientifiques de niveau recherche, publiés ou non, émanant des établissements d'enseignement et de recherche français ou étrangers, des laboratoires publics ou privés. 


\title{
Two-dimensional features of correlations in the flow and near pressure fields of Mach number 0.9 jets
}

\author{
Christophe Bogey* \\ Ecole Centrale de Lyon, Univ Lyon, 69134 Ecully Cedex, France
}

\begin{abstract}
In the present study, two-dimensional spatial correlations are calculated in the flow and the near pressure fields of two isothermal round jets at a Mach number of 0.9, computed by highly-resolved simulations using cylindrical coordinates $(r, \theta, z)$. The two jets have diameterbased Reynolds numbers of 3,125 and 100,000, and they are initially weakly and strongly disturbed, respectively. For both jets, correlations are evaluated between signals at a given point, namely flow fluctuations on the jet axis at the end of the potential core and pressure fluctuations in the jet near field, and 2-D fields acquired in sections $(z, r)$. The full signals but also the axisymmetric and first azimuthal modes are considered. Overall, despite the significant differences in Reynolds number and nozzle-exit conditions, the results for the two jets are very similar. Strong levels of correlations are obtained over large spatial regions and long time periods, providing information on noise generation mechanisms. In particular, the 2-D correlation fields reveal the presence of a wavepacket-like structure growing in the jet mixing layers, centered on a correlation spot in the potential core, and peaking in amplitude around the end of the jet core, which leads to the emission of sound waves in the downstream direction.
\end{abstract}

\section{Introduction}

Since more than sixty years [1, 2], a large amount of studies have been performed to investigate subsonic jet noise. Some questions, however, remain about the low-frequency acoustic component dominating in the downstream direction [3]. This component, typically centered around a Strouhal number of $\mathrm{St}_{D}=f D / u_{j}=0.15$, can be measured over a wide range of Mach numbers [4], typically from $\mathrm{M}=u_{j} / c_{a}=0.5$ up to $\mathrm{M}=2$, where $f$ is the frequency, $D$ and $u_{j}$ are the jet diameter and velocity, and $c_{a}$ is the speed of sound in the ambient medium. It is mainly axisymmetric [5, 6], and has properties which are quite distinct from those of the omnidirectional, broadband noise component prevailing in the upstream and sideline directions, generated by fine-scale turbulence [7]. Therefore, it seems to be produced by large-scale structures and/or instability waves. Furthermore, it appears to be emitted around the end of the potential core [8, 9], by a mechanism which is still not clearly understood, but does not depend much on the Reynolds number [10, 11]. Recently, this mechanism has been assumed to be similar to that predicted in theoretical work on noise generation by hydrodynamic wavepackets [7, 12, 13].

In order to shed light on that mechanism, different methods have been proposed. One method consists in computing cross-correlations between flow quantities and acoustic signals outside the jet. It has been employed since the early seventies in several experimental and numerical investigations [9, 11, 14-20], and very recently for temporallydeveloping jets [21]. It has been found that the noise radiated in the downstream direction correlates with the fluctuations near the end of the potential core for high-subsonic and supersonic jets, suggesting a link between the breakdown of shear-layer turbulent structures and the generation of that noise. The spatial structure and characteristics of the sound field have also been investigated from pressure auto-correlations. The downstream noise component is coherent over large spatial areas, as expected for noise from large-scale structures [7].

In the present work, cross- and auto-correlations are calculated in the flow and the near pressure fields of two isothermal round jets at a Mach number of 0.9 and at Reynolds numbers of 3,125 and 100,000. They are computed from data obtained in highly-resolved simulations in 2-D sections $(z, r)$ of the jets, for the full flow and pressure signals and for the azimuthal modes $n_{\theta}=0$ and 1 alone. Two-dimensional spatial correlations and their time evolutions are thus presented, with the aim of accessing key information on noise generation. Particular attention is paid to the correlations with the fluctuations at the end of the jet potential core, and with the sound pressure fluctuations radiated

*CNRS Research Scientist, Laboratoire de Mécanique des Fluides et d'Acoustique, UMR CNRS 5509, christophe.bogey@ec-lyon.fr, AIAA Senior Member \& Associate Fellow. 
in the downstream direction, in order to further clarify the mechanism underlying the emission of acoustic waves in that direction.

The paper is organized as follows. The characteristics of the jets and of the simulations, including jet initial conditions, numerical methods and computational parameters, are documented, and the two-dimensional cross-correlations are defined in section II. The main properties of the jet flow and near pressure fields, and the correlations are shown in section III. Finally, concluding remarks are given in section IV.

\section{Parameters}

\section{A. Jet flow conditions}

Two isothermal round jets at a Mach number $\mathrm{M}=u_{j} / c_{0}$ of 0.9 and Reynolds numbers $\operatorname{Re}_{D}=u_{j} D / v$ of 100,000 and 3,125 are simulated, where $c_{0}, D$ and $v$ are the speed of sound in the ambient medium, the jet diameter and the kinematic molecular viscosity, as reported in table 1 . The first one has been considered in previous numerical studies [22-24]. The jets originate from a pipe nozzle of radius $r_{0}=D / 2$ and length $2 r_{0}$ into a medium at ambient temperature and pressure $T_{0}=293 \mathrm{~K}$ and $p_{0}=10^{5} \mathrm{~Pa}$. The pipe exit is at $z=0$, and the pipe wall thickness is $0.053 r_{0}$. At the pipe inlet, at $z=-2 r_{0}$, Blasius laminar boundary-layer profiles of thickness $\delta_{B L}=0.15 r_{0}$ for $\operatorname{Re}_{D}=100,000$ and $\delta_{B L}=0.42 r_{0}$ for $\operatorname{Re}_{D}=3,125$ are imposed for the axial velocity [25]. The boundary layer is thinner at a higher Reynolds number, in agreement with experimental trends [26, 27]. In addition, radial and azimuthal velocities are set to zero, pressure is equal to $p_{0}$, and temperature is determined by a Crocco-Busemann relation.

Table 1 Jet parameters: Mach and Reynolds numbers $M$ and $\operatorname{Re}_{D}$, thickness of the Blasius laminar boundarylayer profile imposed at the pipe nozzle inlet $\delta_{B L}$, peak turbulence intensity at the nozzle exit $u_{e}^{\prime} / u_{j}$.

\begin{tabular}{cccc}
\hline $\mathrm{M}$ & $\mathrm{Re}_{D}$ & $\delta_{B L}$ & $u_{e}^{\prime} / u_{j}$ \\
\hline 0.9 & 100,000 & $0.15 r_{0}$ & $9 \%$ \\
0.9 & 3,125 & $0.42 r_{0}$ & $1 \%$ \\
\hline
\end{tabular}

The mean and rms axial velocity profiles obtained at the nozzle-exit section for the two jets are presented in figure 1. The mean velocity profiles are very similar to the laminar profiles imposed at the nozzle inlet, while the rms velocity profiles show peak axial turbulence intensities $u_{e}^{\prime} / u_{j}$ of $9.14 \%$ for $\operatorname{Re}_{D}=100,000$ and of $0.99 \%$ for $\operatorname{Re}_{D}=3,125$. These turbulence intensities are obtained by adding random low-level vortical disturbances uncorrelated in the azimuthal direction at $z=-0.95 r_{0}$ in the boundary layers inside the pipe [22]. This forcing procedure allows us to generate disturbed flow conditions at the nozzle exit, which would otherwise be fully laminar. Thus, the present jets are, respectively, initially highly and weakly disturbed. The nozzle-exit conditions for the jet at $\operatorname{Re}_{D}=100,000$ correspond to those measured in a tripped jet by Zaman [26]. They have been discussed in previous papers [22, 28]. Finally, note that despite the difference in Reynolds number and initial conditions, the two present jets are most likely to radiate noise in a similar manner in the downstream direction [10, 11, 29-31]. For $\operatorname{Re}_{D}=3,125$, however, only large turbulent scales, hence low-frequency sound waves, are expected in the jet flow and acoustic fields which could help us to extract the main features of the noise generation mechanisms associated with the development of coherent structures in a subsonic jet.

\section{B. Numerical methods}

The numerical framework is identical to that used in a number of other jet simulations [22 -25, , 28, 32, 33]. The simulations are carried out using an in-house solver of the three-dimensional compressible Navier-Stokes equations in cylindrical coordinates $(r, \theta, z)$ based on low-dissipation and low-dispersion explicit schemes. The axis singularity is taken into account by the method of Mohseni \& Colonius [34]. In order to alleviate the time-step restriction near the cylindrical origin, the derivatives in the azimuthal direction around the axis are calculated at coarser resolutions than permitted by the grid [35]. For the points closest to the jet axis, they are evaluated using 16 points, yielding an effective resolution of $2 \pi / 16$. Fourth-order eleven-point centered finite differences are used for spatial discretization, and a second-order six-stage Runge-Kutta algorithm is implemented for time integration [36]. A sixth-order eleven-point centered filter [37] is applied explicitly to the flow variables every time step. Non-centered finite differences and filters are also used near the pipe walls and the grid boundaries [25, 38]. At the boundaries, the radiation conditions of Tam 
(a)

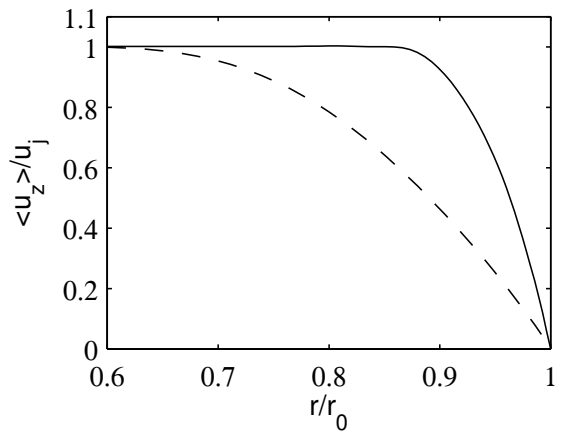

(b)

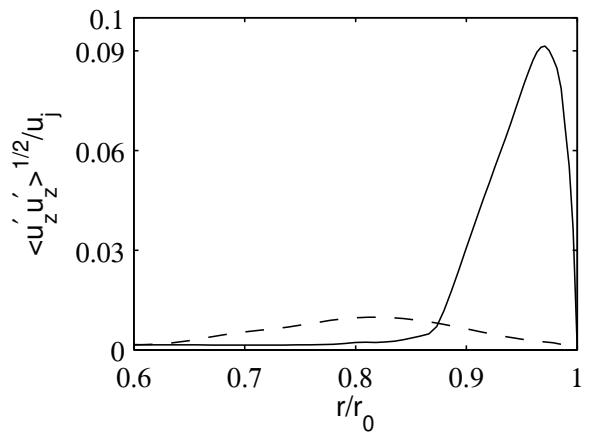

Fig. 1 Nozzle-exit radial profiles of (a) the mean axial velocity $\left\langle u_{z}\right\rangle$ and (b) the rms values of axial velocity fluctuations $u_{z}^{\prime}$ for the jets at $-\operatorname{Re}_{D}=100,000$ and $--\operatorname{Re}_{D}=3,125$.

\& Dong [39] are applied,, with the addition at the outflow of a sponge zone combining grid stretching and Laplacian filtering [40], to avoid significant acoustic reflections. Small adjustment terms are also added to prevent that mean density and pressure deviate significantly from ambient density and pressure, and no co-flow is imposed.

In the present simulation, the explicit filtering is employed to remove grid-to-grid oscillations, but also, when the mesh grid is not fine enough to compute the smallest turbulent structures, as a subgrid-scale high-order dissipation model in order to relax turbulent energy from scales at wave numbers close to the grid cut-off wave number while leaving larger scales mostly unaffected. The performance of this large-eddy simulation (LES) approach has been assessed in past studies for subsonic jets, Taylor-Green vortices and turbulent channel flows [22, 41-44], from comparisons with solutions of direct numerical simulations and from the examination of the magnitude and the properties of the filtering dissipation in the wavenumber space.

\section{Simulation parameters}

The simulation of the jet at $\operatorname{Re}_{D}=100,000$ is a well-resolved LES. Its parameters are detailed in a recent paper [24] reporting the results of a grid-sensitivity study on the jet flow and acoustic fields. The mesh grid contains $n_{r} \times n_{\theta} \times n_{z}=2085 \times 1024 \times 512$ points, and its physical extents are $L_{z}=40 r_{0}$ and $L_{r}=15 r_{0}$. The variations of the radial and axial mesh spacings $\Delta r$ and $\Delta z$ are represented in figure 2 The minimum mesh spacings are equal to $\Delta r=0.0036 r_{0}$ at $r=r_{0}$ and $\Delta z=0.0072 r_{0}$ at $z=0$. The maximal mesh spacing in the jet near field is equal to $\Delta r=0.075 r_{0}$, leading to a Strouhal number of $\mathrm{St}_{D}=f D / u_{j}=5.9$ for an acoustic wave discretized by five points per wavelength, where $f$ is the frequency.

The simulation of the jet at $\mathrm{Re}_{D}=3,125$ is a direct numerical simulation. The mesh grid here is made of $n_{r} \times n_{\theta} \times n_{z}=2458 \times 512 \times 427$ points. It extends, excluding the sponge zone, up to $L_{z}=75 r_{0}$ in the axial direction and out to $L_{r}=25 r_{0}$ in the radial direction. As shown in figure2, the minimum mesh spacings are equal to $\Delta r=0.0072 r_{0}$ at $r=r_{0}$ and $\Delta z=0.014 r_{0}$ at $z=0$, that are twice as large as those for the jet at $\operatorname{Re}_{D}=100,000$. In the jet near field, the maximal mesh spacing is also doubled compared to the higher-Reynolds-number case, with $\Delta r=0.15 r_{0}$ yielding $\mathrm{St}_{D}=3$ for an acoustic wave with five points per wavelength.

The simulations have been performed with an OpenMP-based in-house solver, using a time step $\Delta t$ of $0.7 \times \Delta r(r=$ $\left.r_{0}\right) / c_{0}$ for $\operatorname{Re}_{D}=100,000$ and of $0.6 \times \Delta r\left(r=r_{0}\right) / c_{0}$ for $\operatorname{Re}_{D}=3,125$, to ensure numerical stability. The simulation time $T$ after the transient period is equal to $1,000 r_{0} / u_{j}$ for the jet at $\operatorname{Re}_{D}=100,000$ and to $2,000 r_{0} / u_{j}$ for the jet at $\operatorname{Re}_{D}=3,125$, corresponding to 438,000 and 511,000 iterations, respectively. A total number of approximately 2.5 billion CPU hours has thus been consumed, for $200 \mathrm{~GB}$ of memory required for the higher-Reynolds-number case. During time $T$, density, velocity components and pressure along the jet axis at $r=0$, and on the surfaces located at $r=r_{0}$ and $r=L_{r}$, are recorded at a sampling frequency allowing spectra to be computed up to $\mathrm{St}_{D}=12.8$. Density, velocities and pressure obtained at the azimuthal angles $\theta=0, \pi / 4, \pi / 2,3 \pi / 4, \pi, 5 \pi / 4,3 \pi / 2$ and $7 \pi / 4$ for $\operatorname{Re}_{D}=100,000$ and $\theta=0, \pi / 2, \pi$ and $3 \pi / 2$ for $\operatorname{Re}_{D}=3,125$ are also stored at a halved frequency. Their Fourier coefficients in the azimuthal directions, estimated over the full section $(r, z)$, are also saved for the first nine azimuthal modes. The statistics are averaged in the azimuthal direction, when possible. The time spectra are evaluated from overlapping samples of duration $45 r_{0} / u_{j}$ on the jet axis, and $90 r_{0} / u_{j}$ otherwise. In the azimuthal direction, the 
post-processing is performed up to the mode $n_{\theta}=128$ for $\operatorname{Re}_{D}=100,000$ and $n_{\theta}=64$ for $\operatorname{Re}_{D}=3,125$, where $n_{\theta}$ is the dimensionless azimuthal wave number such that $n_{\theta}=k_{\theta} r$.

(a)

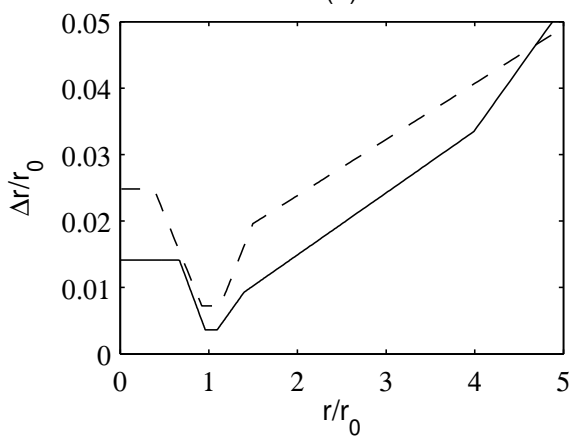

(b)

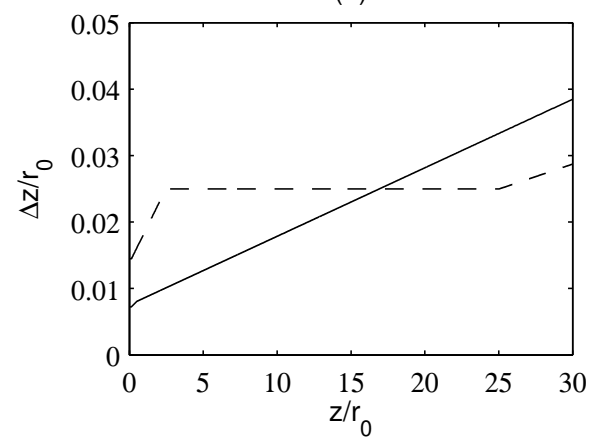

Fig. 2 Variations of (a) the radial mesh spacing $\Delta r / r_{0}$ and (b) the axial mesh spacing $\Delta z / r_{0}$ for the jets at $\longrightarrow \operatorname{Re}_{D}=100,000$ and $--\operatorname{Re}_{D}=3,125$.

\section{Definition of the correlations}

In the present work, two-dimensional spatial correlations are computed in the flow and near pressure fields of the two jets. In practice, a signal $f_{1}$ at a point $r=r_{1}, \theta=\theta_{1}$ and $z=z_{1}$ at time $t$ is correlated with a signal $f_{2}$ in the 2-D section $(r, z)$ at $\theta=\theta_{1}$ at time $t+\delta t$, giving

$$
C(r, z, \delta t)=\left\langle f_{1}\left(r_{1}, z_{1}, t\right) \times g_{2}(r, z, t+\delta t)\right\rangle
$$

where $\delta t$ is the time delay between the two signals, and $\langle$.$\rangle denotes time averaging. Dimensionless correlations$

$$
\mathcal{R}(r, z, \delta t)=\frac{C(r, z, \delta t)}{\left\langle f_{1}^{2}\left(r_{1}, z_{1}, t\right)\right\rangle^{1 / 2} \times\left\langle f_{2}^{2}(r, z, t)\right\rangle^{1 / 2}}
$$

are then evaluated. The signals $f_{1}$ and $f_{2}$ can be the time series of flow and pressure fluctuations issued from the simulations, but also of their Fourier coefficients in the azimuthal direction. The correlations obtained for the different azimuthal angles are averaged, when justified.

In this paper, three types of correlations are examined, depending on the signals $f_{1}$ and $f_{2}$ chosen, as depicted in table 2 In case 1, the fluctuations of axial velocity, vorticity norm and pressure recorded on the jet centerline at $z=z_{c}$, where $z_{c}$ is defined as the position of the end of the potential core, are correlated with the 2-D fluctuating pressure field. In cases 2 and 3 , the pressure fluctuations obtained at $r_{1}=15 r_{0}$, at $z_{1}=40 r_{0}$ and $z_{1}=z_{c}$, respectively, are correlated with the 2-D fields of velocity, vorticity and pressure fluctuations. Therefore, in case 1, the reference 1-D signal is taken in a flow region where the strongest noise sources have been localized experimentally in subsonic jets [8, 45, 46], and where the disturbances have been found to significantly correlate with the downstream noise [9, 11, 16]. In cases 2 and 3, the selected 1-D pressure signals are in the jet near field at $r=15 r_{0}$, where acoustic components are stronger than aerodynamic components according to measurements [47, 48]. In case 2, in particular, the low-frequency strong acoustic waves radiated by the jets in the downstream direction [7] are expected to dominate at the point $\left(r_{1}, z_{1}\right)$ considered [48].

Table 2 Types of two-dimensional spatial correlations.

\begin{tabular}{cccc}
\hline signals & case 1 & case 2 & case 3 \\
\hline$f_{1}\left(r_{1}, z_{1}\right)$ & $u_{z}^{\prime},|\omega|^{\prime}, p^{\prime}$ at $r_{1}=0$ and $z_{1}=z_{c}$ & $p^{\prime}\left(r_{1}=15 r_{0}, z_{1}=40 r_{0}\right)$ & $p^{\prime}\left(r_{1}=15 r_{0}, z_{1}=z_{c}\right)$ \\
$f_{2}(r, z)$ & $p^{\prime}$ & $u_{z}^{\prime},|\omega|^{\prime}, p^{\prime}$ & $u_{z}^{\prime},|\omega|^{\prime}, p^{\prime}$ \\
\hline
\end{tabular}




\section{Results}

\section{A. Jet flow and near pressure field}

\section{Vorticity and pressure snapshots}

Snapshots of the vorticity norm and pressure fluctuations obtained for the two jets are represented in figure 3 Unsurprisingly, the jet flow develops differently depending on the nozzle-exit conditions and Reynolds number. In the initially highly disturbed jet at $\operatorname{Re}_{D}=100,000$, in figure 3 a), the mixing layers contain turbulent structures immediately downstream of the nozzle exit, and very fine scales are visible in the flow. On the contrary, in the initially weakly disturbed jet at $\operatorname{Re}_{D}=3,125$, in figure 3 b), the laminar shear layers roll up around $z=10 r_{0}$, which leads to the formation and pairings of coherent vortical structures. Moreover, only large turbulent scales can be seen due to the effects of viscosity at such a low Reynolds number.

In the same way, the pressure field of the jet at $\operatorname{Re}_{D}=100,000$ exhibits more high-frequency acoustic waves than the jet at $\operatorname{Re}_{D}=3,125$, in particular in the sideline and upstream directions. In both cases, however, large-scale pressure fluctuations, classically attributed to the flow coherent structures [47, 49], emerge in the close vicinity of the jets. In addition, strong low-frequency acoustic waves, typical of the downstream jet noise component [3, 7], propagate in the flow direction for both jets. As in a previous numerical study [31], lowering the jet Reynolds number makes them appear more clearly.

(a)

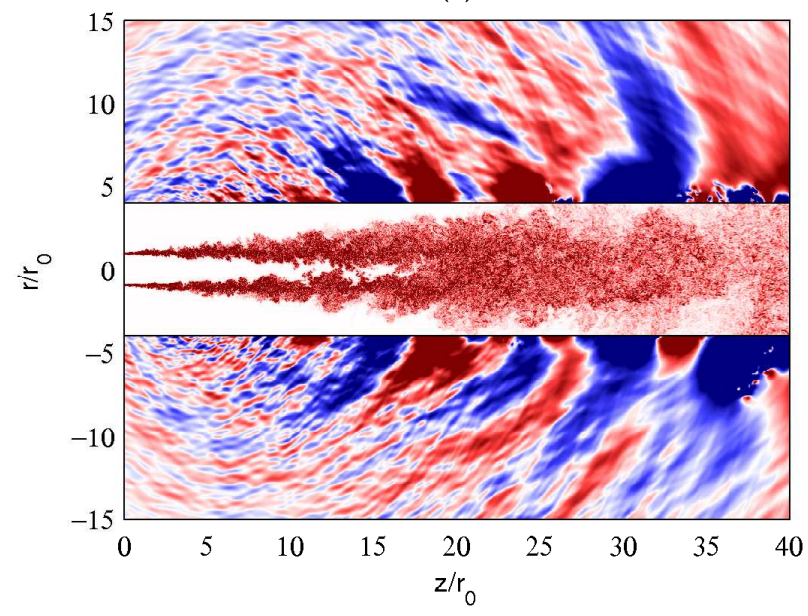

(b)

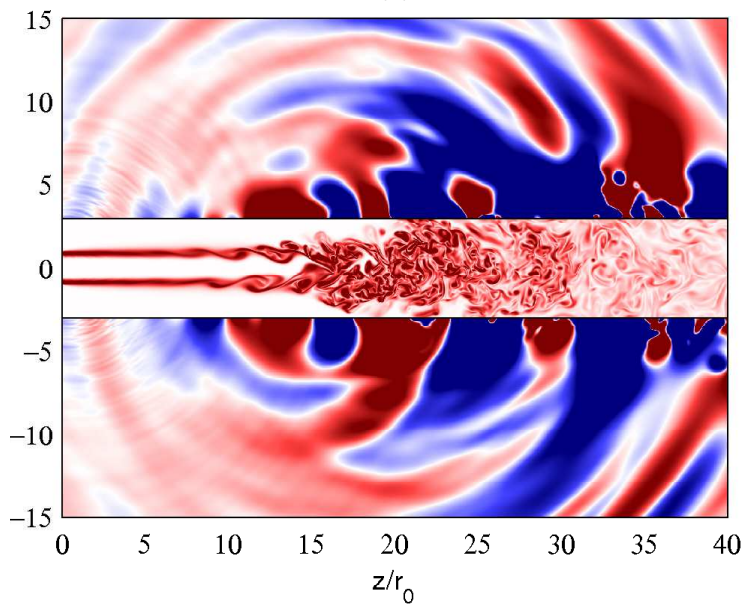

Fig. 3 Representation of vorticity norm inside the flow and of pressure fluctuations outside, for the jets at (a) $\operatorname{Re}_{D}=100,000$ and (b) $\mathrm{Re}_{D}=3,125$. The color scales range between $\pm 6 u_{j} / r_{0}$ and $\pm 70 \mathrm{~Pa}$, from blue to red.

\section{Flow features}

The variations of the shear-layer momentum thickness $\delta_{\theta}$ and of the mean axial velocity $\left\langle u_{z}\right\rangle$ on the centerline for the two jets are presented in figure 4 Experimental data for isothermal jets at $\mathrm{M}=0.9$ and $\operatorname{Re}_{D} \simeq 10^{6}$ are also shown for comparison. In figure 4(a), the mixing layer of the jet at $\mathrm{Re}_{D}=100,000$ is found to spread at a nearly constant rate, similarly to the measurements. On the contrary, the mixing layer of the jet at $\operatorname{Re}_{D}=3,125$ grows slowly from $z=0$ down to $z \simeq 10 r_{0}$, but then very rapidly due to the formation and pairings of coherent structures during the laminar-turbulent transition [50]. These shear-layer developments, caused by two different initial jet flow conditions, are consistent with experimental [51, 52] and numerical [23] results.

In figure 4(b), the velocity profile for the high-Reynolds-number jet is good agreement with the measurements at $\operatorname{Re}_{D} \simeq 10^{6}$. For both jets, coincidentally given the strong discrepancy in shear-layer growth, the velocity on the jet axis starts to decay around $z_{c}=14.6 r_{0}$, where $z_{c}$ is defined as the axial position at which the centerline mean velocity is equal to $0.95 u_{j}$. Farther downstream, the velocity decay is faster for the jet at $\operatorname{Re}_{D}=3,125$ than for the jet at $\operatorname{Re}_{D}=100,000$. This is most likely due to the combined effects of viscosity [41] and laminar nozzle-exit conditions [23] in the former case. 
(a)

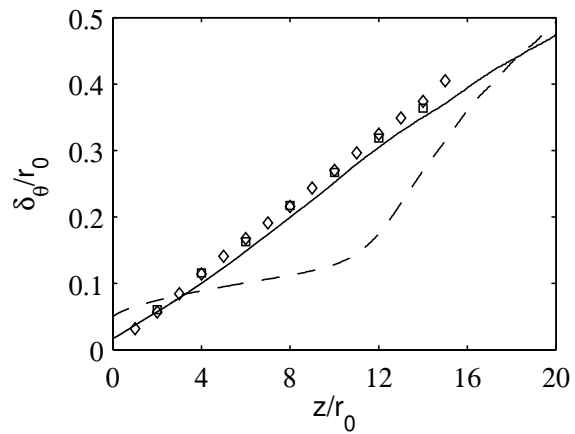

(b)

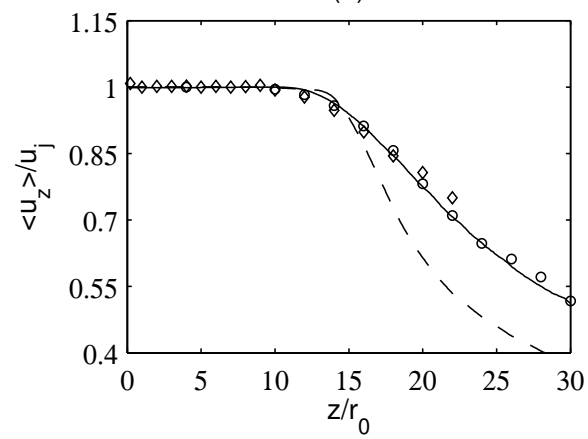

Fig. 4 Variations of (a) the shear-layer momentum thickness $\delta_{\theta}$ and (b) the mean axial velocity $\left\langle u_{z}\right\rangle$ on the centerline for the jets at $-\operatorname{Re}_{D}=100,000$ and $---\operatorname{Re}_{D}=3,125$; measurements for isothermal jets at $M=0.9$ at $\diamond, \square \operatorname{Re}_{D}=10^{6}[53,54]$ and $\diamond \operatorname{Re}_{D}=7.7 \times 10^{5}[55]$.

The variations of the rms and skewness values of axial velocity fluctuations $u_{z}^{\prime}$ along the jet centerline between $z=0$ and $z=30 r_{0}$ are plotted in figure 5. In figure 5(a), the rms velocity profile obtained for the jet at $\operatorname{Re}_{D}=100,000$ compare well with the experimental data of Lau et al. [53] for a jet at at $\operatorname{Re}_{D}=10^{6}$. The peak of turbulence intensities, located a few radii downstream of $z=z_{c}$, is reached slightly farther upstream for the jet at $\operatorname{Re}_{D}=3,125$ than for the other. Above all, the peak is stronger in the initially laminar jet because of the laminar-turbulent transition occurring close to the end of the potential core. This result is in line with previous simulations [23].

Regarding the skewness factor of velocity fluctuations, in figure 5 (b), negative values lower than -1.5 are found near $z=z_{c}$ for both jets. As reported in previous studies [56], including one dealing with temporally developing jets [21], they indicate the intermittent occurrence of velocity deficits on the jet centerline when shear-layer vortical structures intrude into the potential core. This flow feature appeared to be one of the reasons for the significant correlations calculated between velocity fluctuations at the end of the jet core and the low-frequency acoustic waves radiated in the downstream direction [11].

(a)

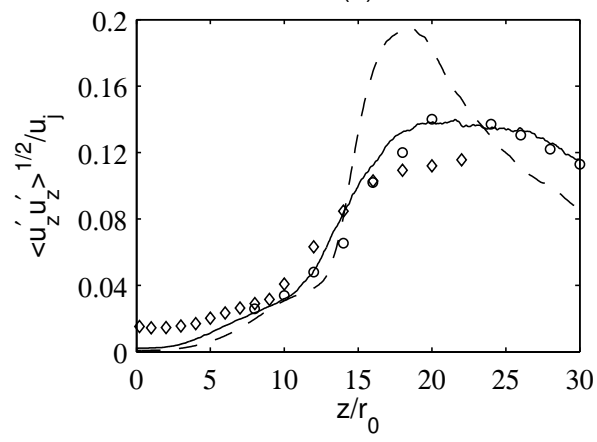

(b)

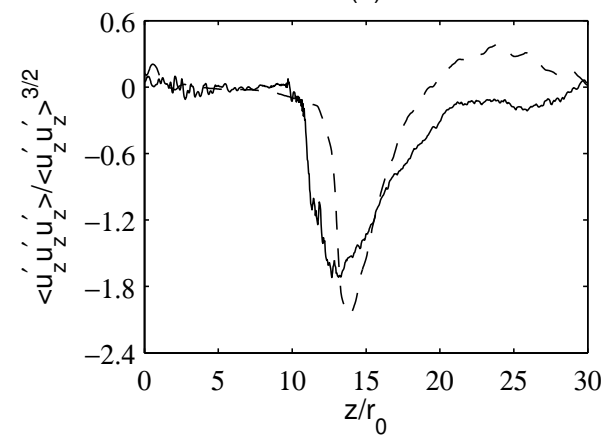

Fig. 5 Variations of (a) the rms values and (b) the skewness factors of the axial velocity fluctuations $u_{z}^{\prime}$ on the centerline for the jets at $-\operatorname{Re}_{D}=100,000$ and $---\operatorname{Re}_{D}=3,125$; measurements for isothermal jets at $M=0.9$ at $\circ \operatorname{Re}_{D}=10^{6}[53]$ and $\diamond \operatorname{Re}_{D}=7.7 \times 10^{5}[55]$.

\section{Near-field pressure spectra}

The pressure spectra obtained in the jet near field at $r=15 r_{0}$ and $z=40 r_{0}$ are first represented in figure 6 (a) as a function of Strouhal number. The one for the jet at $\operatorname{Re}_{D}=100,000$ is in very good agreement with the corresponding measurements [48] for an isothermal jet at $\mathrm{M}=0.9$ and $\mathrm{Re}_{D}=7.9 \times 10^{5}$. As expected, given the low angle of radiation relative to the flow direction, of approximately 30 degrees when taking the end of the potential core as origin, the spectra are both dominated by a low-frequency component centered around $\mathrm{St}_{D}=0.15$ for the jet at $\operatorname{Re}_{D}=100,000$ and $\mathrm{St}_{D}=0.25$ for $\operatorname{Re}_{D}=3,125$. The peak levels are however stronger by $5 \mathrm{~dB}$ for the jet at $\operatorname{Re}_{D}=3,125$, which is 
consistent with the higher turbulence intensities in that case.

The contributions of azimuthal modes $n_{\theta}=0,1$ and 2 to the pressure spectra at $r=15 r_{0}$ and $z=40 r_{0}$ are shown in figure 6(b) for $\operatorname{Re}_{D}=100,000$ and in figure 6(c) for $\operatorname{Re}_{D}=3,125$. For the two jets, for $\mathrm{St}_{D} \leq 0.3$, the contribution of the axisymmetric mode is greater than that of mode $n_{\theta}=1$ by between 3 and $6 \mathrm{~dB}$, which is itself approximately $6 \mathrm{~dB}$ above that of mode $n_{\theta}=2$. A similar prevalence of the axisymmetric mode is found in the far pressure field of jets in the downstream direction [5, 6].

(a)

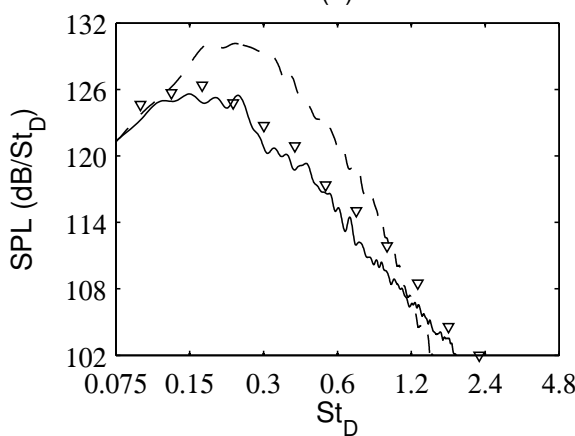

(b)

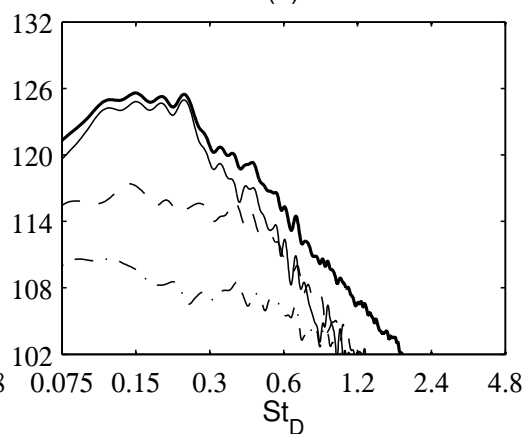

(c)

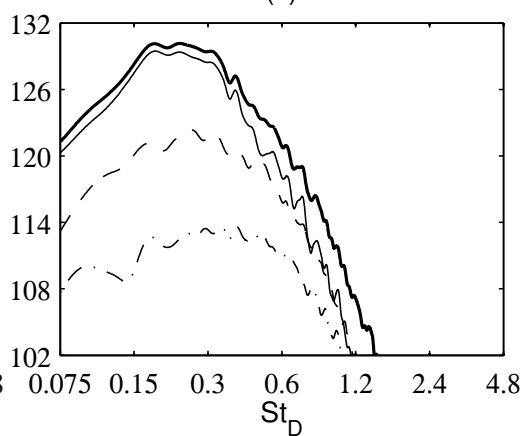

Fig. 6 Sound pressure levels (SPL) obtained at $r=15 r_{0}$ and $z=40 r_{0}$ as a function of $\mathrm{St}_{D}$ : (a) SPL for the jets at $-\operatorname{Re}_{D}=100,000$ and $---\operatorname{Re}_{D}=3,125 ;(b, c)-$ SPL and contributions of modes $n_{\theta}=0,--n_{\theta}=1$ and $-\cdots n_{\theta}=2$ for $(b) \operatorname{Re}_{D}=100,000$ and (c) $\operatorname{Re}_{D}=3,125$; $\nabla$ measurements for an isothermal jet at $M=0.9$ and $\operatorname{Re}_{D}=7.9 \times 10^{5}[48]$.

The pressure spectra obtained at $r=15 r_{0}$ and $z=z_{c}$, perpendicularly to the end of the potential core, are then depicted in figure $7 \mathrm{a}$ ). Compared to those of figure 6 (a), they exhibit levels lower by typically $8 \mathrm{~dB}$, and they are broader. The latter remark is particularly true for the jet at $\operatorname{Re}_{D}=100,000$, whose spectrum has a shape closely following that of the spectrum acquired experimentally [48] at $r=15 r_{0}$ and $z=14 r_{0}$ for a Mach 0.9 jet at a high Reynolds number over the whole frequency range up to $\mathrm{St}_{D}=4.8$. On the contrary, the spectrum for the jet at $\operatorname{Re}_{D}=3,125$ contains negligible high-frequency components for $\mathrm{St}_{D}>1.2$, due to the disappearance of fine-scale turbulence at such a low Reynolds number.

Finally, as previously, the contributions of modes $n_{\theta}=0,1$ and 2 to the pressure spectra are displayed in figure 7 b) for $\operatorname{Re}_{D}=100,000$ and in figure 7 (c) for $\operatorname{Re}_{D}=3,125$. In both cases, no mode emerges clearly, and modes $n_{\theta}=0,1$ and 2 are predominant, respectively, for $\mathrm{St}_{D} \lesssim 0.25$, for $0.25 \lesssim \mathrm{St}_{D} \lesssim 1.2$ and for higher frequencies. A distribution of the acoustic energy between the first three azimuthal modes has also been measured in far field in the sideline direction [5].

(a)

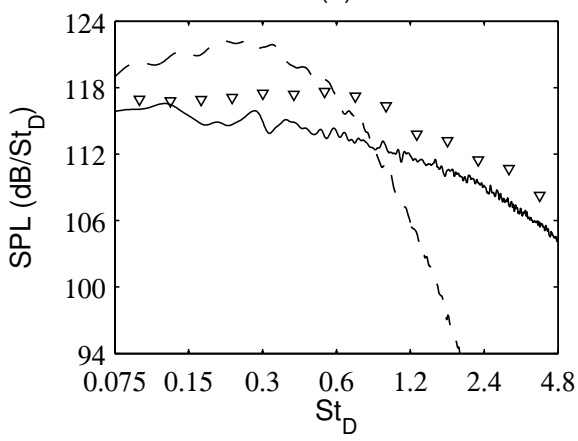

(b)

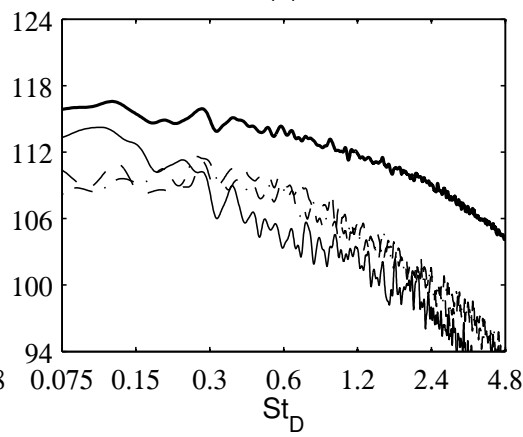

(c)

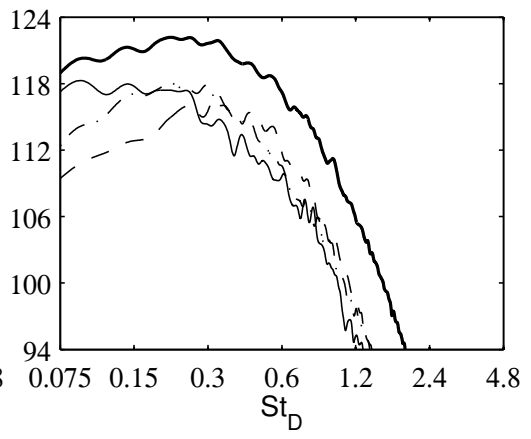

Fig. 7 Sound pressure levels (SPL) obtained at $r=15 r_{0}$ and $z=z_{c}$ as a function of $\mathrm{St}_{D}$ : (a) SPL for the jets at $-\operatorname{Re}_{D}=100,000$ and $---\operatorname{Re}_{D}=3,125 ;(b, c)-$ SPL and contributions of modes $n_{\theta}=0,---n_{\theta}=1$ and $-\cdot-\cdot n_{\theta}=2$ for $(b) \operatorname{Re}_{D}=100,000$ and (c) $\operatorname{Re}_{D}=3,125$; $\nabla$ measurements at $r=15 r_{0}$ and $z=14 r_{0}$ for an isothermal jet at $M=0.9$ and $\operatorname{Re}_{D}=7.9 \times 10^{5}[48]$. 


\section{B. Two-dimensional correlations}

\section{Correlations with fluctuations at the end of the potential core (case 1 in section II.D)}

The normalized correlations $\mathcal{R}$ evaluated between the fluctuations of axial velocity, vorticity and pressure on the jet axis at $z=z_{c}$ and the 2-D fluctuating pressure field for the axisymmetric mode for the jet at $\operatorname{Re}_{D}=100,000$ are represented in figure 8 , from top to bottom. The results obtained for the three time delays of $\delta t=-8 r_{0} / c_{0}, 0$ and $16 r_{0} / c_{0}$ are shown, from left to right. Significant levels of correlations, higher than 0.2 , are found both in the jet and the near pressure fields. For $\delta t=-8 r_{0} / c_{0}$, a wavy structure appears in the flow and its in immediate vicinity, especially in figures $8(\mathrm{a}, \mathrm{g})$. The axial position of this structure, illustrated by a vertical line, indicates a convection at a velocity of approximately $0.7 u_{j}$. The correlation levels increase with time and reach a peak for $\delta t=0$, as expected. At later times, the structure vanishes rapidly, but elongated spots of correlation propagate downstream in the pressure field, see right figures for $\delta t=16 r_{0} / c_{0}$. These spots are roughly aligned with a circle of radius $c_{0} \delta t$ centered on $r=0$ and $z=z_{c}$. They display negative values for the axial velocity in figure 8 (c) and positive values for the vorticity in figure 8(i), which is in accordance with previous studies [11, 21]. The present results reveal the connection between the growth and decay of a large-scale, oscillatory structure in the jet flow and the radiation of a strong acoustic wave in the downstream direction. This mechanism is similar to that predicted in theoretical work on noise generation by hydrodynamic wavepackets in high-speed jets [7, 12, 13], but also to that recently identified for temporal jets using conditional averaging [21].

(a)

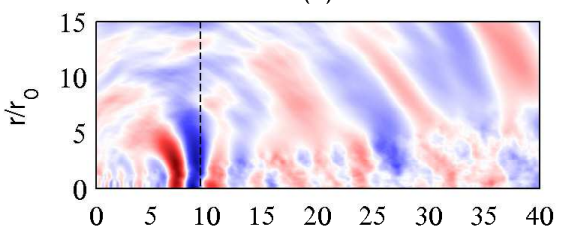

(d)

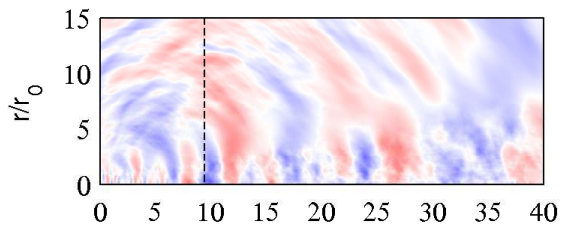

(g)

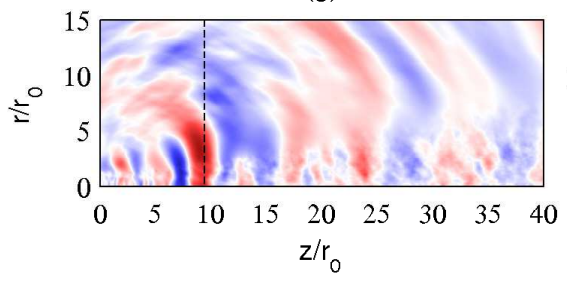

(b)

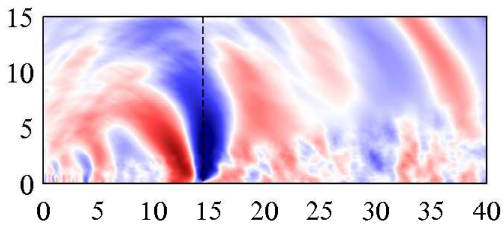

(e)

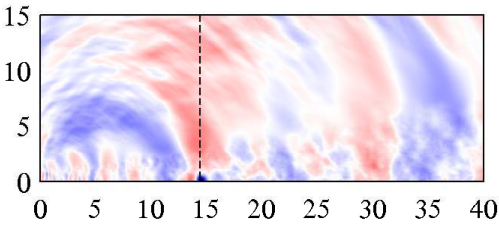

(h)

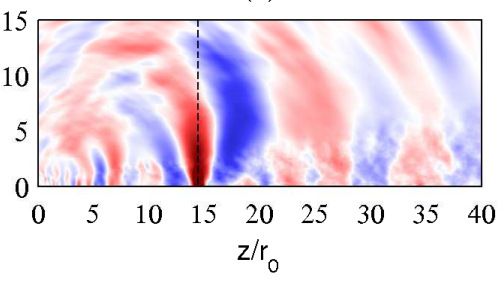

(c)

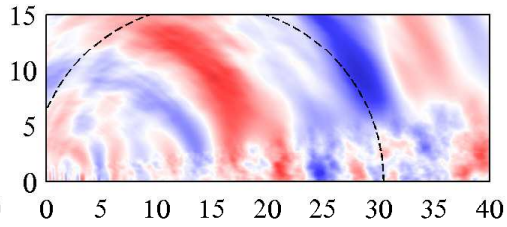

(f)

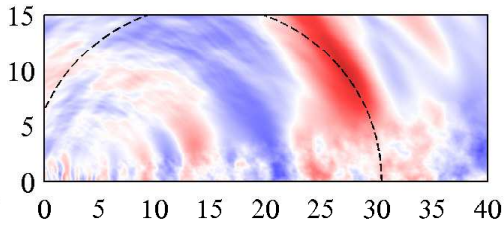

(i)

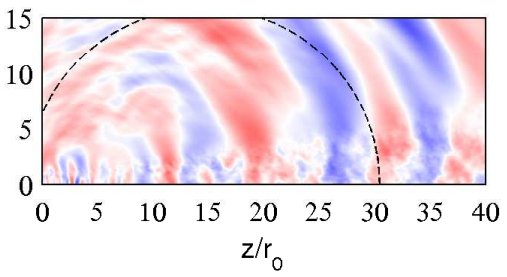

Fig. 8 Jet at $\operatorname{Re}_{D}=100,000$. Correlations $\mathcal{R}$ of (top) $u_{z}^{\prime}$, (middle) $|\omega|^{\prime}$ and (bottom) $p^{\prime}$ at $r=0$ and $z=z_{c}$ with $p^{\prime}(r, z)$ for (left) $\delta t=-8 r_{0} / c_{0}$, (center) $\delta t=0$ and (right) $\delta t=16 r_{0} / c_{0}$, for $n_{\theta}=0$; -- positions for a downstream convection at $0.7 u_{j}$ and a sound propagation at $c_{0}$. The color scale ranges between \pm 0.25 , from blue to red.

As in figure 8 the correlations $\mathcal{R}$ determined between axial velocity, vorticity and pressure at the end of the potential core and the 2-D pressure field for mode $n_{\theta}=0$ for the jet at $\operatorname{Re}_{D}=3,125$ are provided for $\delta t=-8 r_{0} / c_{0}, 0$ and $16 r_{0} / c_{0}$ in figure 9 Compared to the jet at $\operatorname{Re}_{D}=100,000$, they exhibit higher levels, but bear obvious similarities. In particular, the wavepacket-like structure developing and convected at $\sim 0.7 u_{j}$ in the flow is clearly visible for negative time delays. This is also the case for the spots of correlation propagating at a speed of $c_{0}$ in the pressure field for positive time delays. These results further relate the generation the axisymmetric noise component dominant in the downstream direction of jets [5, 6] to a mechanism occurring in the jet core region over a long time and a large spatial extent. They also supports the weak dependence of that mechanism on the Reynolds number [10, 29, 31]. 
(a)

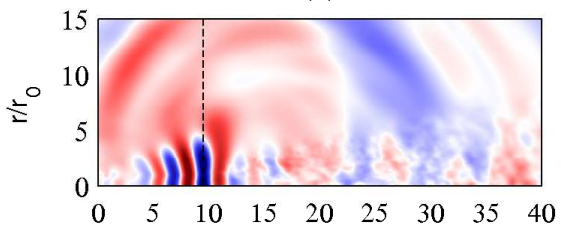

(d)

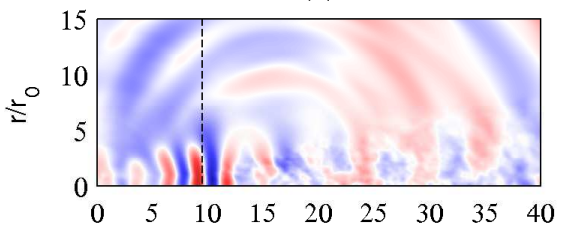

(g)

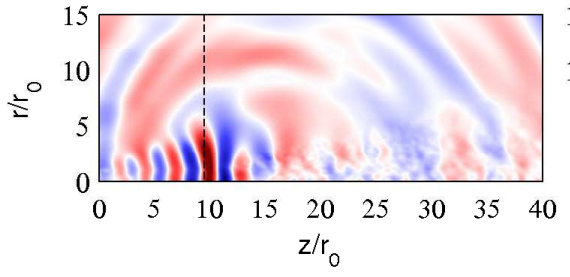

(b)

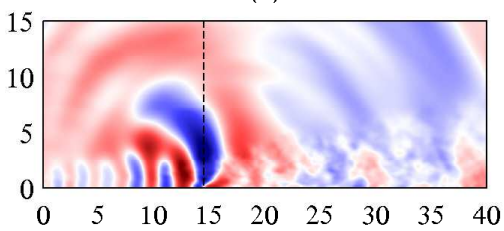

(e)

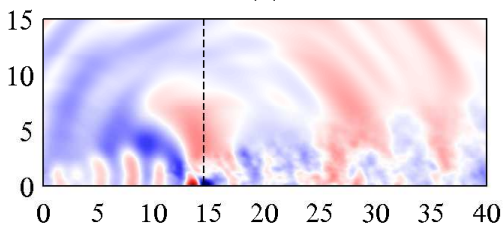

(h)

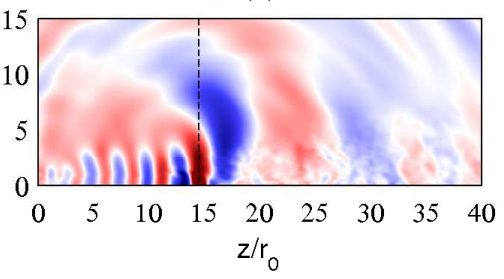

(c)

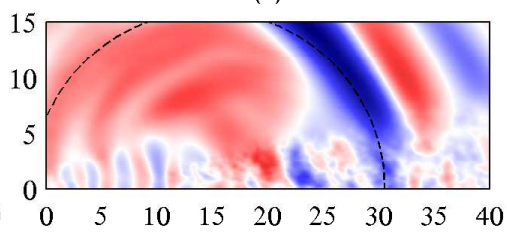

(f)

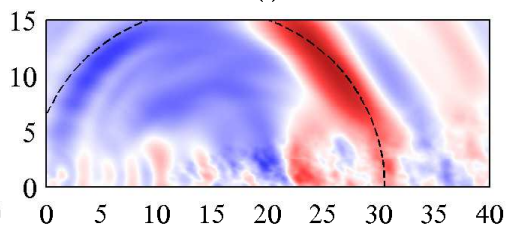

(i)

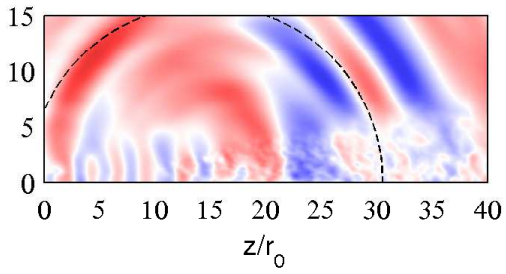

Fig. 9 Jet at $\operatorname{Re}_{D}=3,125$. Correlations $\mathcal{R}$ of (top) $u_{z}^{\prime}$, (middle) $|\omega|^{\prime}$ and (bottom) $p^{\prime}$ at $r=0$ and $z=z_{c}$ with $p^{\prime}(r, z)$ for (left) $\delta t=-8 r_{0} / c_{0}$, (center) $\delta t=0$ and (right) $\delta t=16 r_{0} / c_{0}$, for $n_{\theta}=0$; --- positions for a downstream convection at $0.7 u_{j}$ and a sound propagation at $c_{0}$. The color scale ranges between \pm 0.25 , from blue to red.

\section{Correlations with downstream near-field pressure (case 2 in section II.D)}

The normalized correlations $\mathcal{R}$ obtained between $p^{\prime}$ at $r=15 r_{0}$ and $z=40 r_{0}$ and $p^{\prime}$ in the 2-D section $(z, r)$ from the full signal and the modes $n_{\theta}=0$ and 1 of pressure are represented in figure 10 for the time delay of $\delta t=-12 r_{0} / c_{0}$, from left to right. The results for the jets at $\operatorname{Re}_{D}=100,000$ and 3,125 are provided in the top and bottom figures, respectively. The correlation levels are greater for $\operatorname{Re}_{D}=3,125$ than for $\operatorname{Re}_{D}=100,000$, as expected given the pressure snapshots of figure 3 but also for the axisymmetric mode than for the mode $n_{\theta}=1$. In all cases, however, a front of correlations close to 1 , whose origin seems to be roughly at the end of the jet potential core, and whose minimal distance from the point at $r=15 r_{0}$ and $z=40 r_{0}$ is equal to $c_{0} \delta t$, is observed. This front extends over a very wide range of polar angles, and is surrounded by two fronts of negative correlations. These correlation fields show the high coherence of the jet acoustic components in the downstream direction [7] at both low and high Reynolds numbers [10, 11].

In order to explore the generation of these noise components, the non-normalized correlations $C$ obtained between $p^{\prime}$ at $r=15 r_{0}$ and $z=40 r_{0}$ and the fluctuations of axial velocity in the flow and of pressure outside for the jet at $\operatorname{Re}_{D}=3,125$ are represented in figure 11 for $\delta t=-36 r_{0} / c_{0},-28 r_{0} / c_{0}$ and $-15 r_{0} / c_{0}$, from left to right. For the axisymmetric mode, in the top figures, a wavepacket-like structure grows in the very near pressure field of the jet. Its amplitude is maximum around $\delta t=-28 r_{0} / c_{0}$, when it passes through the end of the potential core, and then becomes weaker. Simultaneously, an acoustic wave is radiated in the jet direction, and gradually deviates from the flow. This process is similar to that revealed in figure 8 for the case 1 of correlations. In the present figure, the wavepacket-like structure is moreover connected to a spot of negative correlations between the downstream near-field pressure and the velocity fluctuations in the flow. This spot develops on the high-velocity side of the mixing layer, and is strongest on the jet axis around the time of sound emission. Such a spot was previously found when the potential core of temporally developing jet closes [21]. It has been attributed to the presence of a velocity deficit when vorticity enters into the jet core.

For the mode $n_{\theta}=1$, in the bottom of figure 11, a wavy structure is convected in the near pressure field surrounding the jet, strengthens down the end of the jet core, and subsequently weakens and radiates sound pressure waves, as for the mode $n_{\theta}=0$. This structure is also attached to a spot of negative pressure-velocity correlations in the flow, which 
(a)

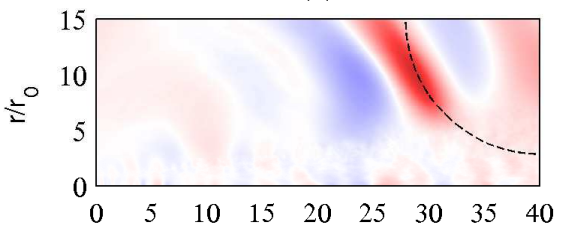

(d)

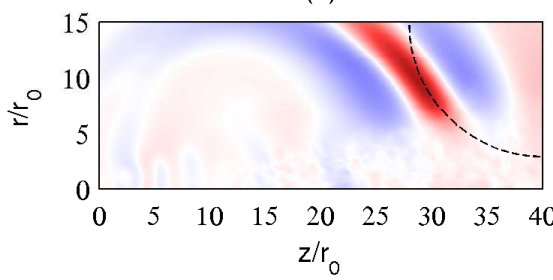

(b)

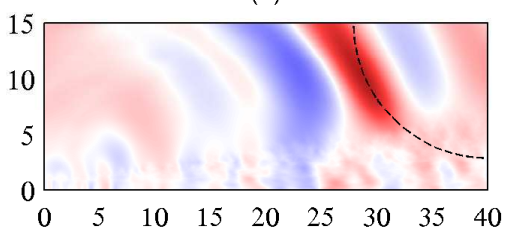

(e)

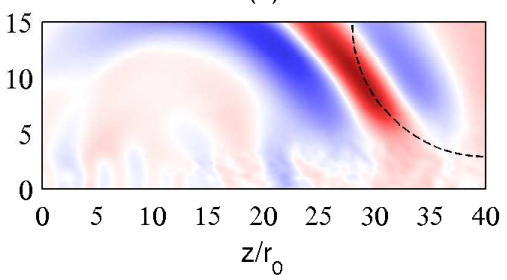

(c)

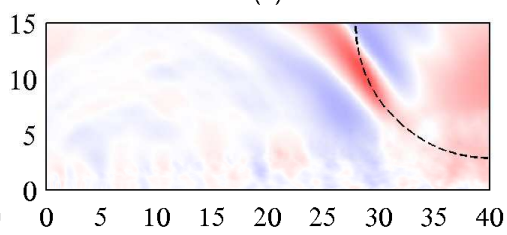

(f)

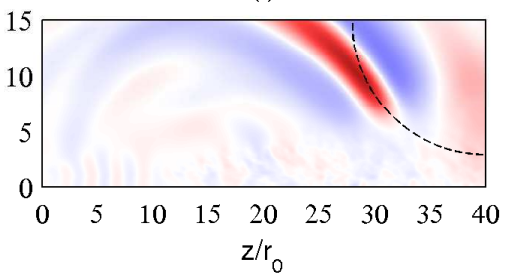

Fig. 10 Correlations $\mathcal{R}$ between $p^{\prime}\left(r=15 r_{0}, z=40 r_{0}\right)$ and $p^{\prime}(r, z)$ for $\delta t=-12 r_{0} / c_{0}$, at (top) $\operatorname{Re}_{D}=100,000$ and (bottom) $\operatorname{Re}_{D}=3,125$ : (left) full field, modes (center) $n_{\theta}=0$ and (right) $n_{\theta}=1$; --- positions for a propagation at $c_{0}$. The color scale ranges between \pm 1 , from blue to red.

are nil on the jet axis in this case. These results suggest that the generation process of the acoustic mode $n_{\theta}=1$ is of the same nature of that of the axisymmetric component in the jet downstream direction.

(a)

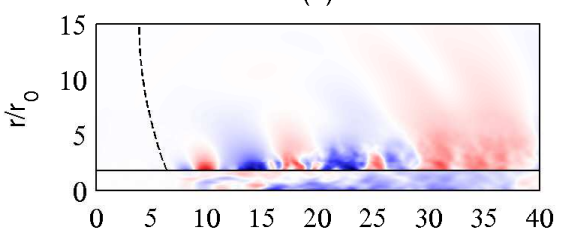

(d)

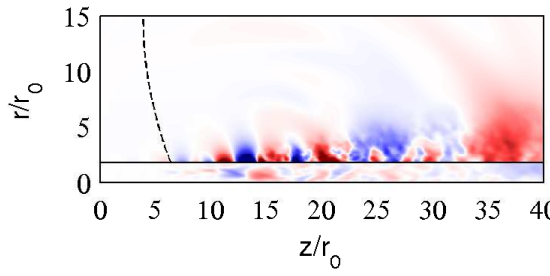

(b)

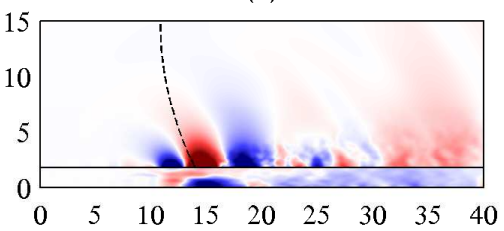

(e)

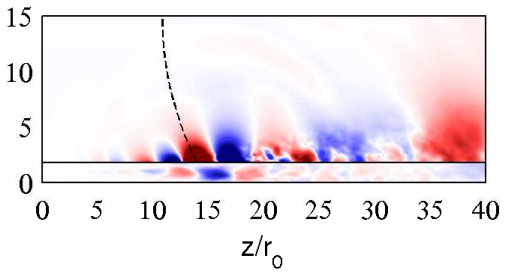

(c)

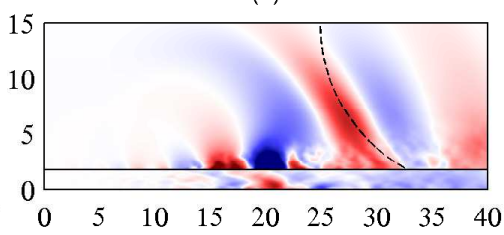

(f)

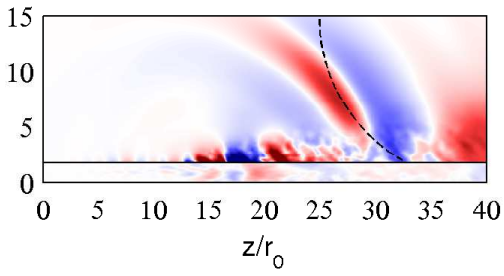

Fig. 11 Jet at $\operatorname{Re}_{D}=3,125$. Correlations $C$ of $p^{\prime}\left(r=15 r_{0}, z=40 r_{0}\right)$ with $u_{z}^{\prime}\left(r \leq 1.75 r_{0}, z\right)$ and $p^{\prime}(r>$ $1.75 r_{0}, z$ ) for (left) $\delta t=-36 r_{0} / c_{0}$, (center) $\delta t=-28 r_{0} / c_{0}$ and (right) $\delta t=-15 r_{0} / c_{0}$, for modes (top) $n_{\theta}=0$ and (bottom) $n_{\theta}=1$ : --- positions for a sound propagation at $c_{0}$. The color scales range between $\pm 0.02 u_{j} \times p_{r m s}^{\prime}$ and $\pm 3 p_{r m s}^{\prime 2}$, from blue to red, where $p_{r m s}^{\prime}$ is the rms value of $p^{\prime}\left(r=15 r_{0}, z=40 r_{0}\right)$.

\section{Correlations with sideline near-field pressure (case 3 in section II.D)}

The normalized correlations $\mathcal{R}$ estimated between $p^{\prime}$ at $r=15 r_{0}$ and $z=z_{c}$ and $p^{\prime}$ in section $(z, r)$ from the full signal and the modes $n_{\theta}=0$ and 1 of pressure are represented in figure 12 for the time delay of $\delta t=-3 r_{0} / c_{0}$, from left to right. As in figure 10, the results obtained for the jets at $\operatorname{Re}_{D}=100,000$ and 3,125 are given in the top and bottom figures, respectively. Significant levels of correlation are found, especially for the axisymmetric mode. They are higher, and extend over a wider region for the lower Reynolds number jet, compare for instance figures 12 (a) and 12 d), indicating that the noise radiated in the sideline direction is more coherent in this case. This results from the fact the sound waves propagating in this direction are generated by large-scale structures developing in the mixing layers for the initially laminar jet at $\operatorname{Re}_{D}=3,125$, whereas they are produced by a broad range of turbulent scales distributed over a 
large axial distance for the initially highly disturbed jet at $\operatorname{Re}_{D}=100,000$, as suggested by the pressure snapshots of figure 3 .

(a)

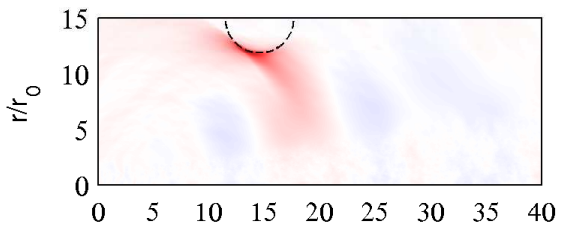

(d)

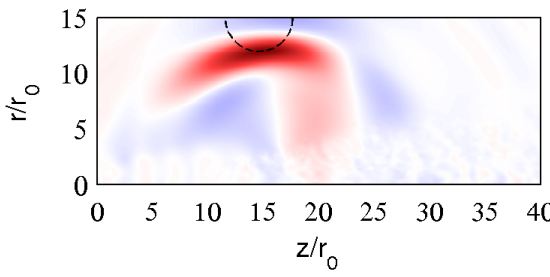

(b)

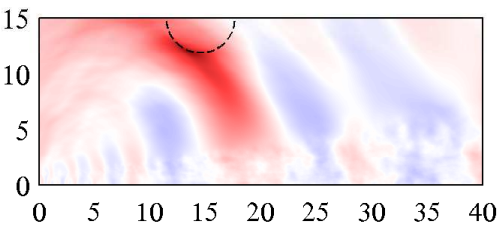

(e)

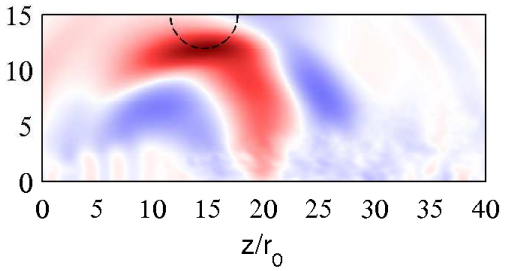

(c)

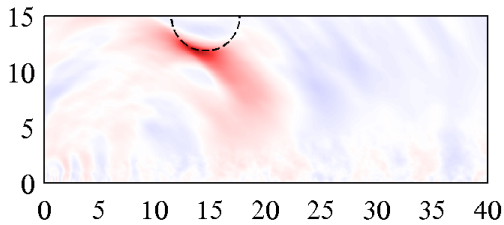

(f)

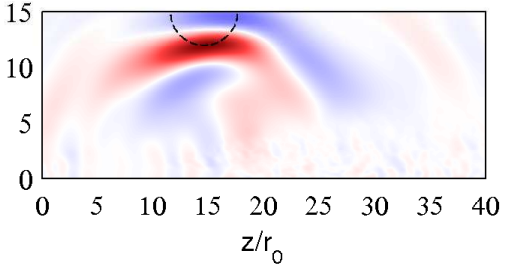

Fig. 12 Correlations $\mathcal{R}$ between $p^{\prime}\left(r=15 r_{0}, z=z_{c}\right)$ and $p^{\prime}(r, z)$ for $t-3 r_{0} / c_{0}$ at (top) $\operatorname{Re}_{D}=100,000$ and (bottom) $\operatorname{Re}_{D}=3$, 125: (left) full field, modes (center) $n_{\theta}=0$ and (right) $n_{\theta}=1$; -- positions for a sound propagation at $c_{0}$. The color scale ranges between \pm 1 , from blue to red.

Finally, the non-normalized correlations $C$ calculated between $p^{\prime}$ at $r=15 r_{0}$ and $z=z_{c}$ and the fluctuations of axial velocity in the flow and of pressure outside for the jet at $\operatorname{Re}_{D}=100,000$ are shown in figure 13 for $\delta t=-15 r_{0} / c_{0}$, $-12 r_{0} / c_{0}$ and $-6 r_{0} / c_{0}$, from left to right. For the axisymmetric mode, on top, the results are roughly similar to those obtained for the correlations with the pressure fluctuations at $r=15 r_{0}$ and $z=40 r_{0}$. Indeed, a wavepacket-like structure, connected to a spot of negative correlations with velocity fluctuations in the flow, is found to be convected and to generate sound pressure waves propagating preferentially in the downstream direction. More surprisingly, positive values of correlation are noted in the jet flow downstream of this spot almost down to $z=40 r_{0}$. For the mode $n_{\theta}=1$, on bottom, a wavepacket-like noise generation mechanism also appears in the correlation fields, albeit less markedly than above. In figure 13 (e), in addition, the correlations are strong over a significant part of the circle of radius $c_{0} \delta t$ centered on the observer point at $r=15 r_{0}$ and $z=z_{c}$, suggesting that they results from several sources in the jet.

(a)

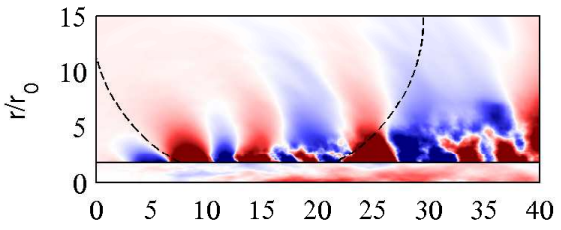

(d)

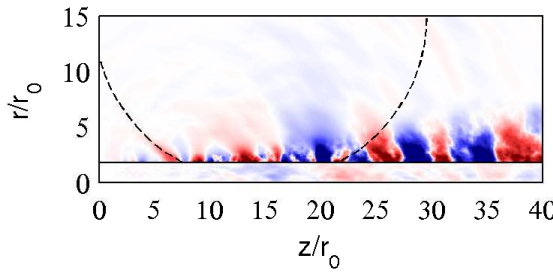

(b)

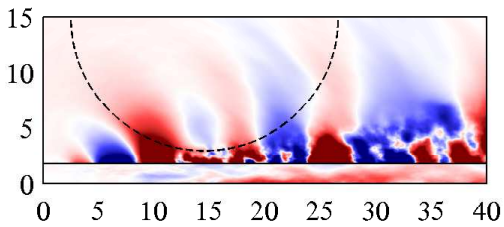

(e)

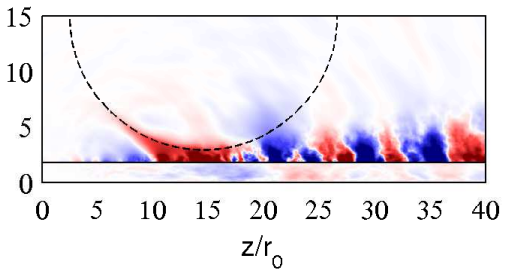

(c)

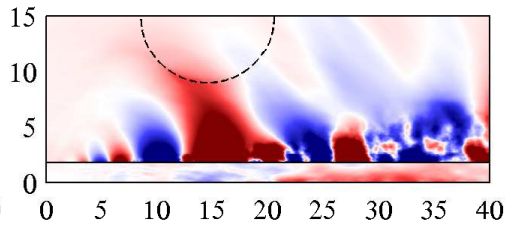

(f)

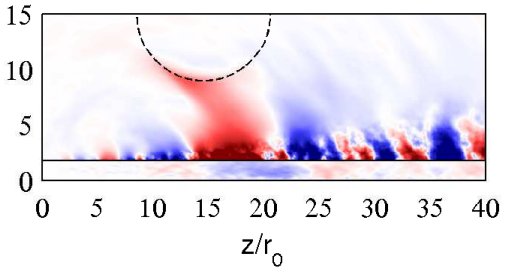

Fig. 13 Jet at $\operatorname{Re}_{D}=100,000$. Correlations $C$ of $p^{\prime}\left(r=15 r_{0}, z=z_{c}\right)$ with $u_{z}^{\prime}\left(r \leq 1.75 r_{0}, z\right)$ and $p^{\prime}(r>$ $\left.1.75 r_{0}, z\right)$ for (left) $\delta t=-15 r_{0} / c_{0}$, (center) $\delta t=-12 r_{0} / c_{0}$ and (right) $\delta t=-6 r_{0} / c_{0}$, for modes (top) $n_{\theta}=0$ and (bottom) $n_{\theta}=1$; -- positions for a sound propagation at $c_{0}$. The color scales range between $\pm 0.02 u_{j} \times p_{r m s}^{\prime}$ and $\pm 3 p_{r m s}^{\prime 2}$, from blue to red, where $p_{r m s}^{\prime}$ is the rms value of $p^{\prime}\left(r=15 r_{0}, z=z_{c}\right)$. 


\section{Conclusion}

In this paper, cross- and auto-correlations calculated in the flow and the near pressure fields of subsonic round jets in sections $(z, r)$ are presented for two jets at a Mach number of 0.9 and at Reynolds numbers of 3,125 and 100,000, with weakly and highly disturbed nozzle-exit boundary layers, respectively. Their two-dimensional structures and time evolutions provide information on the noise radiated by high-subsonic jets and its generation in the jet flows. Concerning the noise emitted in the downstream direction, in particular, strong correlation levels are obtained over very large spatial areas and long time periods. This is true for the pressure auto-correlations in the acoustic field showing the coherence of the sound waves propagating in the jet direction, but also for the cross-correlations computed from aerodynamic disturbances inside and immediately around the flow. In the latter case, a wavepacket-like structure emerges early on in the mixing layers, grows in amplitude as it is convected down to the end of the jet potential core, and then weakens gradually. This structure is connected to a spot of negative correlations between sound pressure and velocity in the flow, but also and especially to the acoustic waves generated in the downstream direction. It can be noted that similar results are found for the full flow and sound fields but also for the modes $n_{\theta}=0$ and 1 taken alone, for both jets. Therefore, the corresponding generation mechanisms appear to be relatively insensitive to the jet Reynolds number and to the initial state and development of the mixing layers. Their characteristics will be explored by further analyses of the present correlation fields.

\section{Acknowledgments}

This work was granted access to the HPC resources of FLMSN (Fédération Lyonnaise de Modélisation et Sciences Numériques), partner of EQUIPEX EQUIP@MESO, and of the resources of CINES (Centre Informatique National de l'Enseignement Supérieur) and IDRIS (Institut du Développement et des Ressources en Informatique Scientifique) under the allocation 2018-2a0204 made by GENCI (Grand Equipement National de Calcul Intensif). It was performed within the framework of the Labex CeLyA of Université de Lyon, operated by the French National Research Agency (Grant No. ANR-10-LABX-0060/ANR-11-IDEX-0007).

\section{References}

[1] Lighthill, M. J., "On sound generated aerodynamically I. General theory,” Proc. Roy. Soc. A, Vol. 211, No. 1107, 1952, pp. 564-587.

[2] Lilley, G. M., "On the noise from air jets," ARC 20, 1958, pp. no. 376-N40-FM 2724.

[3] Mollo-Christensen, E., Kolpin, M. A., and Martucelli, J. R., "Experiments on jet flows and jet noise far-field spectra and directivity patterns," J. Fluid Mech., Vol. 18, No. 2, 1964, pp. 285-301.

[4] Tam, C. K. W., “Jet noise: Since 1952,” Theor. Comput. Fluid Dyn., Vol. 10, No. 1-4, 1998, pp. 393-405.

[5] Juvé, D., Sunyach, M., and Comte-Bellot, G., "Filtered azimuthal correlations in the acoustic far field of a subsonic jet," AIAA J., Vol. 17, No. 1, 1979, pp. 112-113.

[6] Cavalieri, A. V. G., Jordan, P., Colonius, T., and Gervais, Y., "Axisymmetric superdirectivity in subsonic jets," J. Fluid Mech., Vol. 704, 2012, pp. 388-420.

[7] Tam, C. K. W., Viswanathan, K., Ahuja, K. K., and Panda, J., "The sources of jet noise: experimental evidence," J. Fluid Mech., Vol. 615, 2008, pp. 253-292.

[8] Lee, S. S., and Bridges, J., "Phased-array measurements of single flow hot jets," NASA/TM 2005-213826, 2005.

[9] Panda, J., Seasholtz, R. G., and Elam, K. A., "Investigation of noise sources in high-speed jets via correlation measurements," J. Fluid Mech., Vol. 537, 2005, pp. 349-385.

[10] Kastner, J., Samimy, M., Hileman, J., and Freund, J. B., "Comparison of noise mechanisms in high and low Reynolds number high-speed jets," AIAA J., Vol. 44, No. 10, 2006, pp. 2251-2258.

[11] Bogey, C., and Bailly, C., "An analysis of the correlations between the turbulent flow and the sound pressure field of subsonic jets," J. Fluid Mech., Vol. 583, 2007, pp. 71-97.

[12] Suzuki, T., "Review of diagnostic studies on jet-noise sources and generation mechanisms of subsonically-convecting jets," Fluid Dyn. Res., Vol. 42, 2010, p. 014001. 
[13] Jordan, P., and Colonius, T., "Wave packets and turbulent jet noise," Annu. Rev. Fluid Mech., Vol. 45, 2013 , pp. $173-195$.

[14] Lee, H. K., and Ribner, H. S., "Direct correlation of noise and flow of a jet," J. Acoust. Soc. Am., Vol. 52, No. 5, 1972, pp. $1280-1290$.

[15] Seiner, J. M., “The distribution of jet source strength intensity by means of direct correlation technique," Ph.D. thesis, Pennsylvania State University, 81974.

[16] Schaffar, M., "Direct measurements of the correlation between axial in-jet velocity fluctuations and far field noise near the axis of a cold jet," J. Sound Vib., Vol. 64, No. 1, 1979, pp. 73-83.

[17] Panda, J., "Experimental investigation of turbulent density fluctuations and noise generation from heated jets," J. Fluid Mech., Vol. 591, 2007, pp. 73-96.

[18] Bogey, C., Barré, S., Juvé, D., and Bailly, C., "Simulation of a hot coaxial jet: direct noise prediction and flow-acoustics correlations," Phys. Fluids, Vol. 21, No. 3, 2009, p. 035105.

[19] Grizzi, S., and Camussi, R., "Wavelet analysis of near-field pressure fluctuations generated by a subsonic jet," J. Fluid Mech., Vol. 698, 2012, pp. 93-124.

[20] Henning, A., Koop, L., and Schreder, A., "Causality correlation analysis on a cold jet by means of simultaneous Particle Image Velocimetry and microphone measurements," J. Sound Vib., Vol. 332, 2013, pp. 3148-3162.

[21] Bogey, C., "On noise generation in low Reynolds number temporal round jets at a Mach number of 0.9," J. Fluid Mech., Vol. 859, 2019, pp. 1022-1056.

[22] Bogey, C., Marsden, O., and Bailly, C., "Large-Eddy Simulation of the flow and acoustic fields of a Reynolds number $10^{5}$ subsonic jet with tripped exit boundary layers," Phys. Fluids, Vol. 23, No. 3, 2011, p. 035104.

[23] Bogey, C., Marsden, O., and Bailly, C., "Influence of initial turbulence level on the flow and sound fields of a subsonic jet at a diameter-based Reynolds number of $10^{5}$," J. Fluid Mech., Vol. 701, 2012, pp. 352-385.

[24] Bogey, C., "Grid sensitivity of flow field and noise of high-Reynolds-number jets computed by large-eddy simulation," Int. J. Aeroacoust., Vol. 17, No. 4-5, 2018, pp. 399-424.

[25] Bogey, C., and Bailly, C., "Influence of nozzle-exit boundary-layer conditions on the flow and acoustic fields of initially laminar jets," J. Fluid Mech., Vol. 663, 2010, pp. 507-539.

[26] Zaman, K. B. M. Q., "Effect of initial condition on subsonic jet noise,” AIAA J., Vol. 23, No. 9, 1985, pp. $1370-1373$.

[27] Zaman, K. B. M. Q., "Effect of initial boundary-layer state on subsonic jet noise,” AIAA J., Vol. 50, No. 8, 2012, pp. 1784-1795.

[28] Bogey, C., Marsden, O., and Bailly, C., "On the spectra of nozzle-exit velocity disturbances in initially nominally turbulent jets," Phys. Fluids, Vol. 23, No. 9, 2011, p. 091702.

[29] Stromberg, J. L., McLaughlin, D. K., and Troutt, T. R., "Flow field and acoustic properties of a Mach number 0.9 jet at a low Reynolds number," J. Sound. Vib., Vol. 72, No. 2, 1980, pp. 159-176.

[30] Freund, J. B., "Noise sources in a low-Reynolds-number turbulent jet at Mach 0.9," J. Fluid Mech., Vol. 438, 2001, pp. $277-305$.

[31] Bogey, C., and Bailly, C., "Investigation of downstream and sideline subsonic jet noise using Large Eddy Simulation," Theor. Comput. Fluid Dyn., Vol. 20, No. 1, 2006, pp. 23-40.

[32] Bogey, C., Marsden, O., and Bailly, C., "Effects of moderate Reynolds numbers on subsonic round jets with highly disturbed nozzle-exit boundary layers," Phys. Fluids, Vol. 24, No. 10, 2012, p. 105107.

[33] Bogey, C., and Marsden, O., "Identification of the effects of the nozzle-exit boundary-layer thickness and its corresponding Reynolds number in initially highly disturbed subsonic jets," Phys. Fluids, Vol. 25, No. 5, 2013, p. 055106.

[34] Mohseni, K., and Colonius, T., "Numerical treatment of polar coordinate singularities," J. Comput. Phys., Vol. 157, No. 2, 2000, pp. 787-795.

[35] Bogey, C., de Cacqueray, N., and Bailly, C., "Finite differences for coarse azimuthal discretization and for reduction of effective resolution near origin of cylindrical flow equations," J. Comput. Phys., Vol. 230, No. 4, 2011, pp. 1134-1146. 
[36] Bogey, C., and Bailly, C., "A family of low dispersive and low dissipative explicit schemes for flow and noise computations," J. Comput. Phys., Vol. 194, No. 1, 2004, pp. 194-214.

[37] Bogey, C., de Cacqueray, N., and Bailly, C., "A shock-capturing methodology based on adaptative spatial filtering for high-order non-linear computations," J. Comput. Phys., Vol. 228, No. 5, 2009, pp. 1447-1465.

[38] Berland, J., Bogey, C., Marsden, O., and Bailly, C., "High-order, low dispersive and low dissipative explicit schemes for multi-scale and boundary problems," J. Comput. Phys., Vol. 224, No. 2, 2007, pp. 637-662.

[39] Tam, C. K. W., and Dong, Z., "Radiation and outflow boundary conditions for direct computation of acoustic and flow disturbances in a nonuniform mean flow," J. Comput. Acous., Vol. 4, No. 2, 1996, pp. 175-201.

[40] Bogey, C., and Bailly, C., "Three-dimensional non reflective boundary conditions for acoustic simulations: far-field formulation and validation test cases," Acta Acust. united Ac., Vol. 88, No. 4, 2002, pp. 463-471.

[41] Bogey, C., and Bailly, C., "Large Eddy Simulations of transitional round jets: influence of the Reynolds number on flow development and energy dissipation," Phys. Fluids, Vol. 18, No. 6, 2006, p. 065101.

[42] Bogey, C., and Bailly, C., "Turbulence and energy budget in a self-preserving round jet: direct evaluation using large-eddy simulation," J. Fluid Mech., Vol. 627, 2009, pp. 129-160.

[43] Fauconnier, D., Bogey, C., and Dick, E., "On the performance of relaxation filtering for large-eddy simulation," J. Turbulence, Vol. 14, No. 1, 2013, pp. 22-49.

[44] Kremer, F., and Bogey, C., "Large-eddy simulation of turbulent channel flow using relaxation filtering: resolution requirement and Reynolds number effects," Comput. Fluids, Vol. 116, 2015, pp. 17-28.

[45] Chu, W. T., and Kaplan, R. E., "Use of a spherical concave reflector for jet-noise-source distribution diagnosis," J. Acoust. Soc. Am., Vol. 59, No. 6, 1976, pp. 1268-1277.

[46] Fisher, M. J., Harper-Bourne, M., and Glegg, S. A. L., "Jet engine noise source location: The polar correlation technique," J. Sound Vib., Vol. 51, No. 1, 1977, pp. 23-54.

[47] Arndt, R. E. A., Long, D. F., and Glauser, M. N., "The proper orthogonal decomposition of pressure fluctuations surrounding a turbulent jet," J. Fluid Mech., Vol. 340, 1997, pp. 1-33.

[48] Bogey, C., Barré, S., Fleury, V., Bailly, C., and Juvé, D., "Experimental study of the spectral properties of near-field and far-field jet noise," Int. J. Aeroacoust., Vol. 6, No. 2, 2007, pp. 73-92.

[49] Coiffet, F., Jordan, P., Delville, J., Gervais, Y., and Ricaud, F., "Coherent structures in subsonic jets: a quasi-irrotational source mechanism?" Int. J. Aeroacoust., Vol. 5, No. 1, 2005, pp. 67-89.

[50] Winant, C. D., and Browand, R. K., "Vortex pairing : the mechanism of turbulent mixing-layer growth at moderate Reynolds number," J. Fluid Mech., Vol. 63, No. 2, 1974, pp. 237-255.

[51] Browand, F. K., and Latigo, B. O., "Growth of the two-dimensional mixing layer from a turbulent and nonturbulent boundary layer," Phys. Fluids, Vol. 22, No. 6, 1979, pp. 1011-1019.

[52] Husain, Z. D., and Hussain, A. K. M. F., "Axisymmetric mixing layer: influence of the initial and boundary conditions," AIAA J., Vol. 17, No. 1, 1979, pp. 48-55.

[53] Lau, J. C., Morris, P. J., and Fisher, M. J., "Measurements in subsonic and supersonic free jets using a laser velocimeter," $J$. Fluid Mech., Vol. 93, No. 1, 1979, pp. 1-27.

[54] Castelain, T., "Controle de jet par microjets impactants. Mesure de bruit rayonné et analyse aérodynamique," PhD Thesis No. 2006-33, Ecole Centrale de Lyon, France, 2006.

[55] Fleury, V., Bailly, C., Jondeau, E., Michard, M., and Juvé, D., "Space-time correlations in two subsonic jets using dual-PIV measurements," AIAA J., Vol. 46, No. 10, 2008, pp. 2498-2509.

[56] Bogey, C., Bailly, C., and Juvé, D., "Noise investigation of a high subsonic, moderate Reynolds number jet using a compressible LES," Theor. Comput. Fluid Dyn., Vol. 16, No. 4, 2003, pp. 273-297. 